\title{
Transient Ischemic Attack in a High-Risk Cardiovascular Patient with Renal Dysfunction after Treatment with Rivaroxaban and Clopidogrel: A Case Report
}

\author{
Steven J Kary, Caitlin J Roy, William M Semchuk, and Andrea J Lavoie
}

\section{INTRODUCTION}

$\mathrm{P}$

atients with atrial fibrillation who have experienced acute coronary syndrome that was treated with percutaneous coronary intervention (PCI) represent a challenge in antithrombotic management, in terms of balancing the risks of thrombosis and bleeding. The 2018 update of the Canadian Cardiovascular Society's antiplatelet guidelines recommended triple therapy (an oral anticoagulant [OAC], a P2Y12 inhibitor, and acetylsalicylic acid [ASA]) with reduction in the intensity or dose of the OAC and consideration of dual therapy (OAC and P2Y12 inhibitor) within 1 day to 6 months after PCI following acute coronary syndrome. ${ }^{1}$ In contrast, the 2016 atrial fibrillation guidelines of the Canadian Cardiovascular Society recommend triple therapy for 3 to 6 months after PCI in patients with stroke risk defined by a CHADS65 score of 1 or greater. $^{2}$ Triple therapy is associated with a $17.6 \%$ frequency of bleeding requiring hospitalization ${ }^{2}$; dual therapy has been proposed to reduce this risk of bleeding.

The PIONEER-AF-PCI and RE-DUAL-PCI trials assessed the safety of a direct OAC (DOAC) plus a P2Y12 inhibitor relative to the safety of triple therapy., ${ }^{3,4}$ The PIONEERAF-PCI trial ( $n=2124$ patients) demonstrated a reduction in clinically significant bleeding with rivaroxaban $15 \mathrm{mg}$ dual therapy (or rivaroxaban $10 \mathrm{mg}$ for patients with creatinine clearance of $30-50 \mathrm{~mL} / \mathrm{min}$ ) relative to triple therapy $(16.8 \%$ versus $26.7 \%$, respectively; $p=0.002){ }^{3}$ In the RE-DUAL-PCI trial $(n=2725)$, dabigatran dual therapy was associated with a reduction in clinically relevant bleeding relative to triple therapy (for dabigatran $110 \mathrm{mg}, 15.4 \%$ versus $26.9 \%, p<0.001$; for dabigatran $150 \mathrm{mg}, 20.2 \%$ versus $25.7 \%, p<0.001) .{ }^{4}$ Although these safety outcomes are compelling, and there was no signal for loss of efficacy with dual therapy, these trials were underpowered to assess thrombosis outcomes. ${ }^{3,4}$ Furthermore, high-risk populations, including patients with renal dysfunction, were underrepresented in these trials.

The case reported here represents the risks of applying evidence for DOAC dual therapy (with limited data for efficacy) to a high-risk cardiovascular patient with renal dysfunction.

\section{CASE REPORT}

An 82-year-old, 74-kg man with hypertension, dyslipidemia, coronary artery disease treated with PCI (16 years prior), deep vein thrombosis (17 and 21 years prior), and chronic renal insufficiency (baseline serum creatinine $140 \mu \mathrm{mol} / \mathrm{L}$ ) was admitted in May 2017 for elective reverse total shoulder arthroplasty. The evening after surgery, while receiving ASA $325 \mathrm{mg}$ daily, the patient experienced non-ST elevation myocardial infarction and acute-on-chronic renal injury (serum creatinine $185 \mu \mathrm{mol} / \mathrm{L}$ ). A heparin infusion was started, along with clopidogrel $75 \mathrm{mg}$ daily, and the ASA dose was changed to $81 \mathrm{mg}$ daily. New-onset atrial fibrillation was identified on electrocardiography, and amiodarone was initiated. Hematoma of the right shoulder subsequently occurred, and the existing therapy was continued with close observation.

On day 2 after the surgery, the patient exhibited increased confusion and dysphagia; however, the findings of computed tomography (CT) were unremarkable. On day 6, aphasia and right hemiparesis occurred; CT showed an infarct in the left middle cerebral artery of suspected cardioembolic origin. The patient's symptoms recurred throughout the following week, with no changes on repeat CT; the acute renal injury resolved.

*The patient provided consent for publication of this case report. 
On day 15, the patient was transferred to cardiac care for recurrent chest discomfort, with electrocardiography showing significant anterolateral ST depression. Coronary angiography revealed severe left main triple-vessel disease, which was treated with bare metal stents in the left main, ostial circumflex, and obtuse marginal arteries. Therapy with heparin, clopidogrel, and ASA was continued for 3 days after the procedure. On day 20 after the initial shoulder surgery, he was switched to rivaroxaban $10 \mathrm{mg}$ and clopidogrel $75 \mathrm{mg}$ daily, planned to continue for 1 year, as per the recommendations of the PIONEER-AFPCI trial for a patient with creatinine clearance $40 \mathrm{~mL} / \mathrm{min}$ (by weight-based Cockcroft-Gault equation, for serum creatinine $131 \mu \mathrm{mol} / \mathrm{L})(\mathrm{CHADS} 65=4$; bleed risk defined by HASBLED score $=3) .{ }^{3}$ Renal function remained stable after PCI, and the patient did not experience repeat symptoms of stroke. The medications were continued at discharge on day 31 .

On the day after discharge, the patient was readmitted with facial drooping, dysphagia, and right-side hemiparesis; CT showed no significant change. The diagnosis was recurrent transient ischemic attack, which prompted a switch to warfarin, with maintenance of the clopidogrel therapy ( $75 \mathrm{mg}$ daily). The anti-Xa level calibrated for rivaroxaban was not measured, although steady-state drug concentrations were assumed. In follow-up with the neurologist in October 2017, it was noted that the patient continued to have infrequent episodes of aphasia, with subtherapeutic international normalized ratio (INR), following initiation of warfarin. This problem has since resolved, and the patient has not had recurrent stroke or transient ischemic attack, and subsequent INR values have been therapeutic.

\section{DISCUSSION}

The suitability of DOAC dual therapy for this high-risk patient is limited, as his medical conditions were not well represented in the available trials, ${ }^{3,4}$ reducing the external validity of the efficacy outcomes. Given the small subset of patients with renal insufficiency in the trials, initial management with warfarin might have been more appropriate as a well-established option for stroke prophylaxis in patients with atrial fibrillation and declining renal function.

The 2 published trials of DOAC dual therapy would have excluded this patient because of risks of stroke and/or bleeding. More specifically, the PIONEER-AF-PCI trial ${ }^{3}$ excluded patients with a history of stroke; in addition, 254 (35.8\%) of the patients in the dual-therapy experimental arm were 75 years of age or older, and $130(18.5 \%)$ patients had non-ST elevation myocardial infraction. One hundred and ninety-four $(28.8 \%)$ of the patients had creatinine clearance of $30-60 \mathrm{~mL} / \mathrm{min}$; however, data for the subgroup with creatinine clearance of $30-50 \mathrm{~mL} / \mathrm{min}$, who would have received $10 \mathrm{mg}$ rivaroxaban, were not published. The composite rate of death from cardio- vascular causes, myocardial infarction, or stroke was $6.5 \%$ for patients receiving dual therapy versus $6.0 \%$ for those receiving triple therapy, although the study was underpowered to demonstrate significance for this comparison. ${ }^{3}$ The primary composite safety outcome was significantly lower with dual therapy ( $16.8 \%$ versus $26.7 \%, p=0.002$ ), and was driven by bleeding that required medical attention $(13.5 \%$ versus $19.9 \%$, $p=0.001)$ rather than major or minor bleeding. Furthermore, the subgroup analysis of clinically significant bleeding for the rivaroxaban $10 \mathrm{mg}$ group was not published. In the RE-DUALPCI trial, ${ }^{4}$ patients with a stroke in the month before screening were excluded. Patients with previous stroke constituted 11\% of the population, $16 \%$ had renal disease, the indication for PCI was unstable angina for $19.9 \%$ and non-ST elevation myocardial infarction for $20.7 \%$, and the mean stroke risk defined by $\mathrm{CHA}_{2} \mathrm{DS}_{2}$-VASc score was 3.7 in the dabigatran $110 \mathrm{mg}$ group. ${ }^{4}$ The combined dabigatran $110 \mathrm{mg}$ and $150 \mathrm{mg}$ arms demonstrated non-inferiority for the composite end point of thrombosis, death, or unplanned revascularization relative to triple therapy $(13.7 \%$ versus $13.4 \%, p=0.005)$; however, this composite end point was higher for the dabigatran $110 \mathrm{mg}$ $\operatorname{arm}(15.2 \%$ versus $13.4 \%, p=0.30) .{ }^{4}$ The primary composite safety outcome was significantly different between dual and triple therapy (for dabigatran $110 \mathrm{mg}, 15.4 \%$ versus $26.9 \%$, $p<0.001$; for dabigatran $150 \mathrm{mg}, 20.2 \%$ versus $25.7 \%$, $p<0.001$ ), which was maintained for the component of major bleeding (for dabigatran $110 \mathrm{mg}, 5.0 \%$ versus $9.2 \%, p<0.001$; for dabigatran $150 \mathrm{mg}, 5.6 \%$ versus $8.4 \%, p=0.02$ ); however, results for clinically relevant, nonmajor bleeding were not reported. It is unclear whether the outcomes of these trials, in terms of both efficacy and safety, can be extrapolated to similar patients of advanced age with a history of stroke and renal dysfunction.

Future trials may further elucidate the role of DOAC dual therapy. The AUGUSTUS trial (ClinicalTrials.gov identifier NCT02415400, completed November 2018) and the ENTRUST-AF-PCI trial (ClinicalTrials.gov identifier NCT02866175, estimated completion June 2019) are assessing the safety of apixaban and edoxaban dual therapy, respectively. Neither of these trials is assessing efficacy as a primary outcome, and application of their results to high-risk patients, such as the one described here, may be limited.

The patient described here was ultimately treated with warfarin-based dual therapy to balance his high risk of stroke with the risk of bleeding. In patients with high thrombotic risk, elevated bleeding risk, or a complex interplay of these 2 factors, ongoing critical evaluation of recent and emerging evidence is needed to determine optimal antithrombotic therapy.

\section{References}

1. Mehta SR, Bainey KR, Cantor WJ, Lordkipanidzé M, Marquis-Gravel G, Robinson SD, et al. 2018 Canadian Cardiovascular Society/Canadian 
This single copy is for your personal, non-commercial use only.

For permission to reprint multiple copies or to order presentation-ready copies for distribution, contact CJHP at publications@cshp.ca

Association of Interventional Cardiology focused update of the guidelines for the use of antiplatelet therapy. Can J Cardiol. 2018;34(3):214-33.

2. Macle L, Cairns J, Leblanc K, Tsang T, Skanes A, Cox JL, et al. 2016 Focused update of the Canadian Cardiovascular Society guidelines for the management of atrial fibrillation. Can J Cardiol. 2016;32(10):1170-85.

3. Gibson CM, Mehran R, Bode C, Halperin J, Verheugt FW, Wildgoose P, et al. Prevention of bleeding in patients with atrial fibrillation undergoing PCI. N Engl J Med. 2016;375(25):2423-34.

4. Cannon CP, Bhatt DL, Oldgren J, Lip GYH, Ellis SG, Kimura T, et al. Dual antithrombotic therapy with dabigatran after PCI in atrial fibrillation. N Engl J Med. 2017;377(16):1513-24.

Steven J Kary, BSP, ACPR, is with the Saskatchewan Cancer Agency, Saskatoon, Saskatchewan.

Caitlin J Roy, BSP, ACPR, is with the Saskatchewan Health Authority - Regina Area, Regina, Saskatchewan.
William M Semchuk, BSP, MSc, PharmD, is with the Saskatchewan Health Authority - Regina Area, Regina, Saskatchewan.

Andrea J Lavoie, MD, FRCPC, is with the Saskatchewan Health Authority - Regina Area, Regina, Saskatchewan.

Competing interests: Both William Semchuk and Andrea Lavoie have received personal fees from several pharmaceutical companies for work unrelated to the topic of this article. No other competing interests were declared.

Address correspondence to:

Steven J Kary

Saskatoon Cancer Centre

20 Campus Drive

Saskatoon SK S7N 4H4

e-mail: stevenjkary@gmail.com

Funding: None received. 